\title{
Energy-Efficient Clustering WSN System for Environment Monitoring Applications
}

\author{
Amer Abu Salem ${ }^{1}$, Tareq Alhmiedat ${ }^{2}$ \\ ${ }^{1}$ Zarqa University, Jordan,abusalem@zu.edu.jo \\ ${ }^{2}$ Tabuk University, KSA, t.alhmiedat@ut.edu.sa
}

\begin{abstract}
Air pollution is considered critical to people's comfort, health and safety. In both environments (indoor and outdoor) air pollution can be controlled using a small amount of inexpensive sensing units distributed across an area of interest for measuring the levels of different critical gasses such as $\mathrm{CO}, \mathrm{H}_{2} \mathrm{~S}$ and $\mathrm{NO}_{2}$. The monitoring process is critical. The deployment of the Wireless Sensor Network offers an alternative solution by scattering a large number of disposable sensor nodes across an area of interest. Scientists may directly retrieve sensed data via a web server application from the sensor area. This paper offers an extensive review of the approaches available to support the environmental monitoring of the wireless sensor network.
\end{abstract}

Key words: Environment Monitoring; Wireless Sensor Networks; Communication Protocols

\section{INTRODUCTION}

Clean air is a fundamental requirement for everyday life; air pollution affects human health and is regarded as a major global problem, particularly in countries with a ubiquitous gas and petroleum industry. Air quality is defined by calculating such pollutants, such as carbon monoxide (CO), surface level ozone $\left(\mathrm{O}_{3}\right)$, and hydrogen sulfide $\left(\mathrm{H}_{2} \mathrm{~S}\right)$, which affect human safety, according to the United States Environmental Protection Agency (U.S. EPA) [1].

It is not only the main aim of environmental monitoring to collect information from a number of sites, but also to provide research, planning and policy-makers with the knowledge necessary to enable them to make decisions on environmental management and development, as well as to provide end-users with useful information.Habitat and weather tracking represent a major class of sensor network technologies.

There are tremendous efforts to improve air quality in both environments: indoors and outdoors. Recent developments in low-power wireless network technologies also provided the technological conditions for building small, multi-functional sensor tools to track and control physical phenomena. A wide range of uses include military, medical, environmental monitoring, health, and civil science [2, 3, and 4]. Wireless Sensor Networks (WSNs) are now an active area of research. Several definitions of WSNs are already described and produced for different purposes in the literature.
The main concept of WSN environmental monitoring systems is illustrated in Figure 1. The paper addresses several technical issues to be discussed before designing and implementing the environmental monitoring program, including the right topology of the wireless network, the contact protocol, the selection of environmental sensors and the main platforms. The research in [5] includes the exterior WSN environmental surveillance systems. Nevertheless, in accordance with the study of indoor and greenhouse monitoring systems, this paper provides up to date surveys.

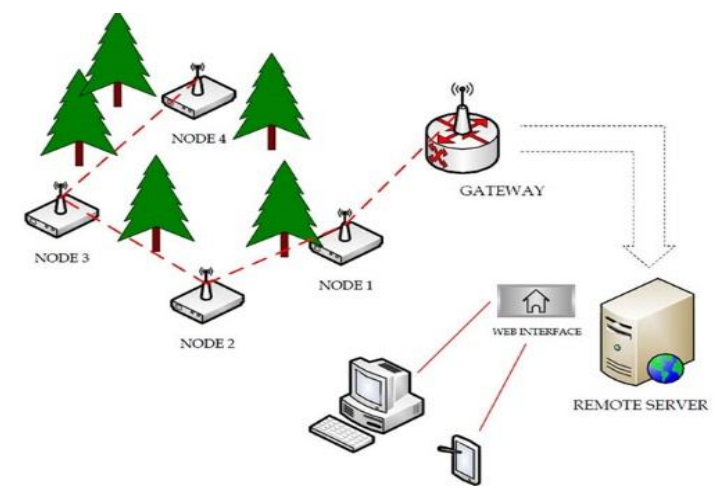

Figure 1: Environment monitoring using sensor networks

The majority of this paper is structured accordingly. Section 2 lays out the word WSN, while Section 3 addresses the procedures for the coordination with WSN. Section 4 discusses the recently developed current WSN environmental surveillance systems. A paper evaluating the current WSNbased environmental monitoring programs is introduced at Section 5 although problems and weaknesses. The key needs for the design and implementation of a WSN-based environmental surveillance system and concludes the work outlined in this paper.

\section{WIRLESS SENSOR NETWORK}

A WSN is a sensor node array distributed in a cooperative network. Sensor nodes are low, cost-intensive and have a short range of communication. Each WSN's basic component is the sensor node. Depending on the complexity of the individual sensor nodes, the expense for sensor nodes ranges from few to hundreds of dollars.

In general, a sensor node consists of four sub-systems as shown in Figure 2:

- A computing system: responsible for key functions such as communication protocol processing and on-board sensor power. 
- A sensing system: characteristics of the environment are sensed by various sensors (temperature, humidity, light, gas, etc.).

- A module for communication: a limited radio range to connect with nearby nodes.

- A system for power supply: this includes a battery source that supplies computing, on-board sensors and communication systems.

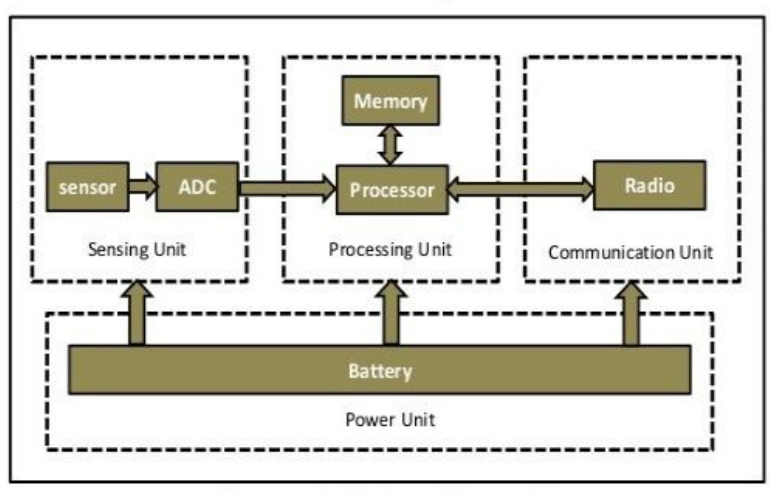

Figure 2: Sensor node architecture

\section{ENVIRONMENT MONITORING SYSTEMS}

Many research groups are working on the design and development of WSN based environmental monitoring systems. In this section, the existing WSN based environment monitoring systems are summarized and discussed, in which they are categorized based on deployment area into three main categories; indoor, outdoor, and greenhouse, as presented in Figure 3.

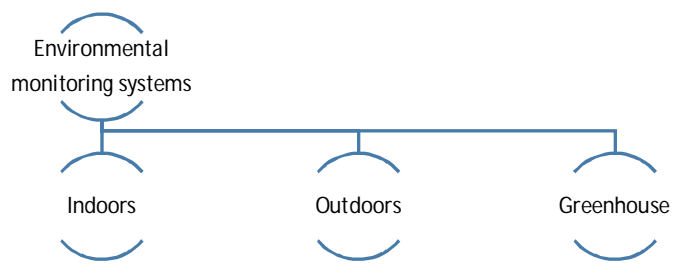

Figure 3: Classification of WSN environmental monitoring systems

The indoor environmental monitoring systems are discussed. Essentially, pollution needs to be considered indoors (homes, offices, and buildings). Indoor pollutants may be created through indoor activities including cooking and smoking. Authors of [6] stated that indoor air quality is generally assessed by measuring the concentration of $\mathrm{CO}$, temperature, and humidity. The work presented in [7] includes the design and development of an indoor WSN real time monitoring system, which consists of a desktop computer acting as a server, web-based interface program, and sensing module with an array of sensors including $\mathrm{CO}_{2}$, particle matter, temperature, and humidity. In [8] authors developed a wireless solution for indoor air quality monitoring, to measure the environmental parameters (temperature, humidity, gaseous pollutants), and determine the environmental health of an indoor space, which was represented in terms of Air Quality Index (AQI). The work presented in [9] includes a WSN for monitoring indoor air quality, which is significant for people's comfort, health, and safety. A system with an aggressive energy management system was presented at the sensor level, node level, and the network level. The sensor node contains a metal oxide semiconductor gas sensor and pyroelectric infrared (PIR) sensor, which detects the presence of people in certain places. Authors of [10] proposed a prototype of a WSN for monitoring the air quality of an arbitrary indoor infrastructure environment, which specifically monitors the level of $\mathrm{CO}_{2}$ levels indoors. On the other hand, a WSN based industrial monitoring system was proposed in [11], to monitor temperature, humidity, and gas. Real experiments were conducted using arm7 embedded microprocessor and XBee modules. A low cost indoor environmental pollution gas monitoring system was implemented in [12], where its main goal was to measure the concentrations of COand $\mathrm{CO}_{2}$ gases. The proposed system is fast response, requires low maintenance, user friendly, and has ability to achieve real time measurements. SensorScope [13] is a collaborative project between environmental and network researchers, which aims at providing an efficient and inexpensive out-ofthe-box WSN-based environmental monitoring system. Authors addressed the problem of energy by developing an efficient data gathering algorithm to reduce the power consumption for tiny sensor nodes. A WSN-based environmental monitoring system for air quality measurement and natural gas leakages is proposed in [14]. The proposed system is based on catalytic off-the shelf available gas sensors and on a new strategy of sampling and processing, which offers low power consumption. The proposed system has been tested using Mics-5121 sensor for measuring the level of carbon monoxide and volatile organic compounds, and Jennic modules as transmission mechanism. As presented above, various types of sensor arrays were deployed in the indoor environmental monitoring systems, however most of the existing systems measure the concentration of COand $\mathrm{CO}_{2}$ gases, which mainly contaminates the indoor air quality, and threating human health and safety. Therefore, there was a great demand to study the impact of those gases through adopting the indoor environmental monitoring systems.

Second, the outdoorenvironmental monitoring systems are discussed. Outdoor air quality can be expressed by measuring the concentration of several pollutants, including $\mathrm{CO}$, Ozone $\left(\mathrm{O}_{3}\right)$, Sulphur dioxide $\left(\mathrm{SO}_{2}\right)$, and nitrogen dioxide $\left(\mathrm{NO}_{2}\right)$ [6] Since the effect of air pollution on human health is considered a major and serious problem globally, therefore the design and development of environmental monitoring systems is necessary. In [6] authors proposed a WSN-based measuring system for air quality monitoring using tin dioxide sensor arrays connected to an acquisition and control system. Two architectures were proposed for wireless communication between the sensing nodes and a personal computer that manages the whole system. An ambient real-time air quality monitoring system was proposed in [15], in which it consists of several distributed monitoring stations which communicate wirelessly with a backend server using machine-to-machine communication. Each stationary node is equipped with gaseous and meteorological sensors, in addition to data logging and wireless communication capabilities. A number of 4 stationary sensor nodes were deployed over an area of 1 $\mathrm{km} 2$. The server collects real time data from stationary sensor nodes and converts it into information delivered to users through web portals and mobile applications. SECOAS [16] is an environmental monitoring system deployed to observe the impact of a newly developed wind arm on coastal 
processes in the area at Scroby sands off the coast of Great Yarmouth. New sensor hardware was developed based on MCU PIC 18F452, where each sensor node is equipped with pressure, turbidity, temperature, and salinity sensors. Data transferred from sensor nodes to a base station, and end-user can access data from land station via Internet connection. A depth study of applying WSN to real world habitat monitoring is presented in [17], and includes a tired system architecture, in which the lowest layer consists of 32 nodes distributed on a small island off the coast of Maine, whereas the higher layer includes a gateway and web-servers. ASWP [18] is a long-term WSN deployment for environmental monitoring, which has been running for two years, and implements a periodic sampling application for external sensors exposed to a forested outdoor environment in western Pennsylvania, USA. The proposed system studied the packet duplication, packet loss, and network maintenance. Authors of [19] designed and implemented an online General Packet Radio Service (GPRS) sensors array for air pollution monitoring, which consists of a mobile data acquisition unit, and a fixed Internet enabled pollution monitoring server. The data-acquisition unit includes single-chip microcontroller, air pollution sensors array, GPRS-modem, and a Global Positioning System (GPS) module. The Mobile dataacquisition unit gathers air pollutants levels $\left(\mathrm{CO}, \mathrm{NO}_{2}\right.$, and $\mathrm{SO}_{2}$ ), and packs them in a single frame with the GPS location, time and date. The frame is then uploaded to the pollution server using a GPRS-modem. P-Sense [20] is a WSN air pollution monitoring and control system, where its main goal was to allow government officials, international organizations, communities, and individuals access to the pollution data to address their particular troubles and need. PSense provides large amounts of pollution data in time and space with different granularities. The work presented in [21] proposed a marine environmental monitoring framework based on WSN technology, characterized by energy harvesting, robustness with respect to a large number of sensor nodes. The developed system is based on Ad-Hoc through star topology, in which it consists of sensor nodes with local transmission, and control center and data storage for real-time visualization. In [22], authors proposed a WSN framework, designed to observe the impacts of climate change in crop fields. The prototype system consists of two base stations and several sensor nodes which are powered by solar cell panel. The sensed climate parameters from sensor nodes are sent through WSN to the base station, where base station communicates with remote data server through GPRS/3G network. However, in this work, the power consumption was validated using NS-2 simulation tool. WAPMS [23] refers to WSN Air Pollution Monitoring System; its main goal was to monitor air pollution in Mauritius through the use of sensor nodes deployed in large areas around the island. Through this work, a new data aggregation algorithm was proposed, named Recursive Converging Quartiles (RCO), which is used to merge data to remove duplicates, filter out incorrect readings, and thus saving energy. The proposed system has been validated using Jist/Swans simulator. Authors of [24] proposed a WSN based environment monitoring systems based on ZigBee and GPRS technologies as communication protocols. Authors stated that pollution map can be built using off-shelf sensor equipment, and the experimental results may be published using web services such as Google Maps. In [25], authors proposed a WSN based framework to monitor changes in environmental conditions. A number of 25 base stations distributed over 5 countries using infield sensor data collected from vineyards. End-users may interactively access the sensed data through a web application. A wireless mesh network environmental monitoring system was proposed in [26]. The system collects and stores the $\mathrm{SO}_{2}, \mathrm{NO}_{2}$, and $\mathrm{NO}$ concentration, in addition to measure the level of temperature, humidity, and air pressure. The proposed system was tested using ARM9 S3C2410 that improves the processing speed and reduces the power consumption. Numerous projects with real implementations have focused on the outdoor environmental monitoring. The most widespread array of sensors deployed in the outdoor environmental monitoring systems are: temperature, humidity, $\mathrm{CO}, \mathrm{CO}_{2}$, and $\mathrm{O}_{2}$.

Third, the greenhouseenvironmental monitoring systems are considered. A greenhouse is a building where plants are grown. Greenhouse protects crops from high heat or cold, in addition to shield plants from storms. A number of WSNbased greenhouse environmental monitoring systems have been proposed recently. In [27], authors presented a low cost and energy efficient prototype of a wireless environment monitoring system, for observing greenhouse gases including $\mathrm{CO}, \mathrm{CO}_{2}, \mathrm{SO}_{2}, \mathrm{NO}_{2}$, and $\mathrm{O}_{2}$. The prototype consists of the communication module, the wireless smart transmitter interface, and wireless network capable application processor modules. These modules have been successfully developed using the IEEE802.15.4, IEEE1451.2, and IEEE1451 standards. A greenhouse monitoring system was proposed in [28], where its main goal was to observe the pepper vegetable surrounding environment. The proposed system includes the development of a WSN application for precision agriculture aimed to improve the quality in agricultural production, and minimize the management and farming costs. An online WSN based greenhouse gases monitoring system proposed in [29], and was implemented using XBee module and Arduino microcontroller. A number of three nodes were deployed in the experiment test-bed, in which sensors data are sent directly to a central monitoring unit that acts as a sink node, and attached to a personal computer. Authors of [30] designed a low cost greenhouse monitoring system to monitor a temperature and humidity parameters, and adopt the ZigBee technology as a communication protocol between sensor nodes. A simple sensor network system consists of 2 nodes placed at the agriculture department Melaka Tengah greenhouse in Malaysia, to test the feasibility of the designed system. In [31] authors proposed a WSN based embedded system and deals with the implementation of ZigBee protocol for remote controlling of the greenhouse parameters. The proposed system consists of two subsystems: portable controller node $(\mathrm{PCN})$ and sensor and actuator node (SAN). The former consists of user laptop and XBee module, which collects the sensed data from the SAN nodes and display the gathered data on a GUI Java-based system that facilitates real time monitoring of various greenhouse gases, whereas SAN consists of an array of sensors including temperature, humidity, soil, light, and moisture, in which these sensors sense the greenhouse environment and transmit the sensed values to PCN. A real-time WSN greenhouse monitoring system was proposed in [32] that monitors important factors in the plant growth such as temperature, humidity, and carbon monoxide concentration. The implemented system consists of three parts: sensing module, radio communication module and gateway module, where the real-time implementation has 
been conducted in a Harumanis Mango greenhouse which is located at UniMAPAgrotechnology Research Station in Perlis, Malaysia. The work presented in [33] involves the design of a simple, easy to install, and a microcontrollerbased circuit to monitor and record the values of temperature, humidity, and soil moisture of the natural environment, in order to achieve maximum plant growth yield based on ZigBee protocol. Deploying manual systems in monitoring the greenhouse field threats the production rate. Temperature and humidity levels of the greenhouse must be constantly monitored to ensure the optimal conditions. The common sensors deployed in the existing greenhouse monitoring systems are: temperature, humidity, $\mathrm{CO}$ and $\mathrm{CO}_{2}$.

\section{DISCUSSION}

Many research groups are working on the design and development of WSN based environmental monitoring systems, each solution differs in the type of sensors used, communication protocol between sensor nodes, cost, and power consumption. In this section, a comparison is presented, in which the existing systems are studies and compared. Table summarizes the most representative WSN based environmental monitoring systems developed recently, and have been studied in terms of the following aspects:

\section{Experiment's Size and Type}

Simulation experiments [9, 22, and 23] are unable to capture the complexity of outdoor environments, and affect the WSN functionalities. Therefore, real-world experimental deployments are necessary to study and analyze their harsh conditions. On the other hand, it's necessary to study the number of sensor nodes deployed in the real experiment, in order to validate the system's efficiency and applicability.

\section{Environmental Sensors Array}

Clean air is the basic requirement of daily life. People are exposed to air pollutants in both environments indoors and outdoors. Therefore, to detect the level of pollution in a specific environment, a proper array of sensors is required to be installed to measure the concentration of critical gases such as: $\mathrm{CO}_{2}, \mathrm{CO}, \mathrm{NO}_{2}$, and $\mathrm{SnO}_{2}$.

\section{Communication Protocol between Sensor Nodes}

The communication protocol plays a significant role in WSN applications. Sensor nodes need to communicate to each other via a cost and power efficient communication protocol, to make the data available at the sink node.

\section{Data Transmission Way to a Web-server}

End-user including the scientist and policy makers may retrieve the sensed data from a web-server. Sensor nodes transmit the sensed data to a sink node, then sink node may upload the received data to a web server.

\section{Power Consumption}

Gas sensors are power-hungry, and sensor nodes must operate unattended for several years on a battery power supply. Therefore, it is essential to consider the power consumption issue when developing a WSN based environmental monitoring system. For evaluation purposes, power consumption was estimated based on the communication protocol deployed, and the types of environmental sensor array used.

\section{Cost per node}

In environmental monitoring applications, a large number of sensor nodes are required to be scattered over an area of interest. Cost is estimated based on the types of environment sensors used, the transmission module, and the integration of any additional devices, such as GPS receiver, and/or GPRS modem.

\section{Platform}

This includes devices and tools deployed to implement the environmental monitoring systems. Since, various number of development kits available on market with various costs and power consumption.

\section{THE CHALAENGES}

This section reviews the most relevant challenges for the existing WSN environmental monitoring systems, in which the limitations of the existing systems lie on the following aspects:

\section{Power Consumption}

Power consumption is an essential issue for long-term operation. Environmental monitoring systems are considered as high power consumption, since gas sensors are energy hungry [9]. In the outdoor environmental monitoring systems, sensor nodes are placed unattended for long time on a battery power supply. The systems presented in $[6,26]$ are inefficient in terms of power consumption, because they were implemented using IEEE 802.11 communication protocol, whereas the system presented in [20] was practically tested using Bluetooth communication protocol. Both IEEE 802.11 and Bluetooth are considered as ineffective energy consumption protocols for environmental monitoring systems. One the other hand, several Environmental monitoring systems integrated high power consumption devices such as GPS and GPRS technologies [15, 19, 20, and 24].

\section{Scalability}

Environmental sensor nodes must be easy to deploy and maintain in large environments. Bluetooth and PRS based systems [6, 20, and 26] face the problem of low scalability. As stated above, Bluetooth network can support up to 7 slave devices, whereas GPRS technology is based on the cellular network's GSM band to transmit data. The availability of mobile network towers may be low in certain locations (i.e. forests), where the environmental monitoring system is installed.

\section{Sensor Node Cost}

Cost is a significant factor when the environmental monitoring system is required to be installed in a wide area, where a large number of sensor nodes are required to be deployed. Additional cost is presented when adopting energy harvesting methods such as solar panels $[13,15]$. On the other hand, a number of existing systems [6, 19] use the technology of the GPRS as a main communication way between sensor nodes, which consequently offers high cost 
per node. Also the systems presented in $[18,19]$ depend on a high cost localization method (i.e. GPS technology).

\section{WSN Communication Protocol}

A number of wireless communication protocols are available recently, such as IEEE 802.15.4, ZigBee, and Wireless HART. The IEEE 802.15.4 represents a milestone in standardization efforts. The systems presented in $[6,18,19$, $26]$ have been implemented using inefficient communication protocols: Bluetooth and IEEE 802.11.

\section{THE REQUIREMENTS}

After considering an extensive study on the existing environmental monitoring system, a number of requirements have to be taken into consideration before designing and implementing a WSN environmental monitoring system. The requirements for such solutions are as follows:

\section{Low Cost of Deployment}

In environmental monitoring applications, sensor networks are meant to be deployed in thousands of nodes to achieve wider coverage. Therefore, the sensor nodes are required to be easy to deploy and low in cost. Available wireless and sensor platforms on the market are expensive. Hence, produce cheaper sensor node platform is a requirement.

\section{Scalability}

In environmental monitoring applications, WSN can accommodate thousands of nodes. The existing WSN environmental systems employed tens to hundreds nodes. Therefore, it is essential to prove that the available theoretical solutions are suited to large real WSN.

\section{Power Efficient}

This is critical for long-term operation, especially when it is required to monitor remote and hostile environments. Data aggregation, clustering methods, cross layer protocols, and new power storage devices are presented as possible solutions to improve the sensor nodes lifetime.

\section{Positioning}

Sensor nodes are required to be localized in order to determine the geographical location of pollution. Therefore, there is a significant requirement to employ a light localization algorithm for WSNs [26, 27].

\section{Remote Access}

End-users require accessing the data available from sensor nodes over certain geographical area. Besides using WSN communication protocols (such as ZigBee and

Wireless HART), sensor nodes may use different other mains of radio communications to send data to the gateway (webserver) where sensed data are stored.

\section{CONCLUSION}

WSNs have recently received quite attention in the context of environmental monitoring as presented in the literature. In both environments indoors and outdoors, people are exposed to air pollutants emitted by houses, equipment, factories, vehicles, and many others. This paper targets the WSN based environmental monitoring research area. Wide varieties of such systems have been developed and implemented recently, driven by the need to obtain air quality estimation with the minimum cost possible. In this paper, a review is provided which discusses the existing environment monitoring systems. This paper points out several research-oriented problems that need to be addressed before environmental monitoring applications can be effectively implemented in practice.

\section{ACKNOWLEDGMENT}

The authors would like to acknowledge the financial support for this work, from the Deanship of Scientific Research, Zarqa University, Zarqa, Jordan.

\section{REFERENCES}

[1] The United States Environmental Protection Agency (US EPA), http://www.epa.gov/.

[2] T. Alhmiedat, F. Omar, A. Abu Taleb, \& A. Alsswey."Road Safety and Energy Saving Proposed System: A Zigbee WSN Approach."International Journal of Online Engineering (iJOE). Vol. 11, No. 2 (2015): pp-55.

https://doi.org/10.3991/ijoe.v11i2.4430

[3] S. Nouha, A. Mellouk, B. Augustin, Y. Amirat, J. Marty, M. Khoussa, A. Abid, \& R. Zitouni. "Wireless Sensor Networks for medical care services." In WirelessCommunications and Mobile Computing Conference (IWCMC), pp. 571-576. IEEE, 2011.

[4] L. Louise, M. Toulgoat, M. Deziel, \& G. Patterson. "Tiered wireless sensor network architecture for military surveillance applications", The Fifth International Conference on Sensor Technologies and Applications, pp. 288-294. 2011.

[5] L. Oliveira \& J. Rodrigues. "Wireless sensor networks: a survey on environmental monitoring." Journal of communications. Vol. 6, No. 2 (2011): pp. 143-151.

https://doi.org/10.4304/jcm.6.2.143-151

[6] O. A. Postolache, J. M. Pereira, \& P. M. B. Girao. "Smart sensors network for air quality monitoring applications." IEEE Transactions on Instrumentation and Measurement, 58, no. 9: 3253-3262. 2009.

[7] S. Saad, A. Shakaff, A. Saad, \& A. Yusof. "Development of wireless sensor network for monitoring indoor air pollutant." In International Conference On Mathematics, Engineering And Industrial Applications (Icomeia 2014), vol. 1660, p. 070077. AIP Publishing, 2015.

https://doi.org/10.1063/1.4915795

[8] S. Bhattacharya, S. Sridevi, \& R. Pitchiah. "Indoor air quality monitoring using wireless sensor network." In Sensing Technology (ICST), 2012 Sixth International Conference on, pp. 422-427. IEEE, 2012.

[9] V. Jelicic, M. Magno, D. Brunelli, G. Paci, \& L. Benini. "Context-adaptive multimodal wireless sensor network for energy-efficient gas monitoring." IEEE Sensors Journal, Vol. 13, no. 1: 328-338, 2013.

https://doi.org/10.1109/JSEN.2012.2215733

[10] P. Spachos, L. Song, \& D. Hatzinakos. "Prototypes of opportunistic wireless sensor networks supporting indoor air quality monitoring." In Consumer Communications and Networking Conference (CCNC), 2013 IEEE, pp. 851-852. IEEE, 2013. 
[11] T. Rajuu, R. Sekhar, N. Raju, \& P. Kumar. "WSN Based Industrial Environmental Monitoring System Using uC/OS 11" International Journal of Research in Computer and Communication Technology, Vol 2. No. 12. 2013.

[12] A. Kumar, I. P. Singh, \& S. K. Sud. "Indoor environment gas monitoring system based on the digital signal

processor." International Conference On Multimedia, Signal Processing and Communication Technologies. IMPACT'09, pp. 245-249. 2009.

[13] G. Barrenetxea, F. Ingelrest, G. Schaefer, M. Vetterli, O. Couach, \& M. Parlange. "Sensorscope: Out-of-the-box environmental monitoring." International Conference on Information Processing in Sensor Networks, 2008. IPSN'08, pp. 332-343. IEEE, 2008.

[14] M. Rossi, \& D. Brunelli. "Ultra low power wireless gas sensor network for environmental monitoring applications." In Environmental Energy and Structural Monitoring Systems (EESMS), 2012 IEEE Workshop on, pp. 75-81. IEEE, 2012. https://doi.org/10.1109/EESMS.2012.6348397

[15] A. Kadri, E. Yaacoub, M. Mushtaha, \&A Abu-Dayya. "Wireless sensor network for real-time air pollution monitoring." 1st International Conference on Communications, Signal Processing, and their Applications (ICCSPA), pp. 1-5. IEEE, 2013.

[16] M. Britton, \& L. Sacks. "The SECOAS Project: development of a self-organising, wireless sensor network for environmental monitoring." In Second International Workshop on Sensor and Actor Network Protocols and Applications. 2004.

[17] A. Mainwaring, J. Polastre, R. Szewczyk, D. Culler, \& J. Anderson. "Wireless sensor networks for habitat monitoring." In Proceedings of the 1st ACM international workshop on Wireless sensor networks and applications, pp. 88-97. ACM, 2002.

https://doi.org/10.1145/570738.570751

[18] M. Navarro, T. W. Davis, Y. Liang, \& X. Liang. "A study of long-term WSN deployment for environmental monitoring." IEEE 24th International Symposium on Personal Indoor and Mobile Radio Communications (PIMRC), 2013, pp. 2093-2097. IEEE, 2013.

[19] A. R. Ali, I. Zualkernan, \& F. Aloul. "A mobile GPRSsensors array for air pollution monitoring." IEEE Sensors Journal, Vol. 10, no. 10: 1666-1671. 2010.

[20] D. Mendez, A. J. Perez, M. Labrador, \& J. Jose Marron. "P-sense: A participatory sensing system for air pollution monitoring and control." In Pervasive Computing and Communications Workshops (PERCOM Workshops), IEEE International Conference on, pp. 344-347. IEEE, 2011.

[21] C. Alippi, R. Camplani, C. Galperti, \& M. Roveri. "A robust, adaptive, solar-powered WSN framework foraquatic environmental monitoring." IEEE Sensors Journal Vol. 11, no. 1 (2011): 45-55.

https://doi.org/10.1109/JSEN.2010.2051539

[22] T. Nguyen, T. Thanh, L. Nguyen, \& H. Huynh. "On the design of energy efficient environment monitoring station and data collection network based on ubiquitous wireless sensor networks." In Computing \& Communication TechnologiesResearch, Innovation, and Vision for the Future (RIVF), 2015 IEEE RIVF International Conference on, pp. 163-168. IEEE, 2015.

[23] K. Khedo, R. Perseedoss, \& A. Mungur. "A wireless sensor network air pollution monitoring system."
International Journal of Wireless \& Mobile Networks (IJWMN), Vol. 2. No. 2. May, 2010.

[24] A. Bagula, M. Zennaro, G. Inggs, S. Scott, \& D. Gascon. "Ubiquitous sensor networking for development (usn4d): An application to pollution monitoring." Sensors 12, no. 1: 391414. 2012.

https://doi.org/10.3390/s120100391

[25] A. Ghobakhlou, S. Zandi, \& P. Sallis. "Development of environmental monitoring system with wireless sensor networks." 19th International Conference on Modelling and Simulation, Perth, Australia, Dec. 2011.

[26] M. Gao, F. Zhang, \& J. Tian. "Environmental monitoring system with wireless mesh network based on embedded

system." In Fifth IEEE International Symposium on Embedded Computing. SEC'08, pp. 174-179. IEEE, 2008.

[27] A. Kumar, \& G. P. Hancke. "Energy efficient environment monitoring system based on the IEEE 802.15. 4 standard for low cost requirements." IEEE Sensors Journal, Vol. 14, no. 8: 2557-2566, 2014.

https://doi.org/10.1109/JSEN.2014.2313348

[28] M. Srbinovska, C. Gavrovski, V. Dimcev, A. Krkoleva, \& V. Borozan. "Environmental parameters monitoring in precision agriculture using wireless sensor networks." Journal of Cleaner Production Vol. 88: 297-307. 2015.

[29] M. A. Reddy, \& V. Natarajan, "On-line Monitoring of Green House Gases Storage and Leakage using Wireless Sensor Network", International Research Journal of Engineering and Technology (IRJET), Vol. 2. No. 1. 2015.

[30] A. Salleh, M. Ismail, R. Mohamad, M. Abd Aziz, M. Othman, \& M. Misran. "Development of greenhouse monitoring using wireless sensor network through ZigBee technology." International Journal of Engineering Science Invention (IJESI) 2, no. 7 (2013): 6-12.

[31] J. Baviskar, A. Mulla, A. Baviskar, S. Ashtekar, \& A. Chintawar. "Real time monitoring and control system for green house based on 802.15.4 wireless sensor network." Fourth International Conference on In Communication Systems and Network Technologies (CSNT), pp. 98-103. IEEE, 2014.

https://doi.org/10.1109/CSNT.2014.28

[32] S. Saad, L. Kamarudin, K. Kamarudin, W. Nooriman, S. Mamduh, A. Zakaria, A. Shakaff, \& M. Jaafar. "A realtimegreenhouse monitoring system for mango with Wireless Sensor Network (WSN)." 2nd International Conference on In Electronic Design (ICED), pp. 521-526. IEEE, 2014.

[33] V. Katti, \& A. Pise. "Remote monitoring of Greenhouse Parameters using Zigbee Wireless Sensor Network." In International Journal of Engineering Research and Technology, vol. 3, no. 2 (February-2014). ESRSA Publications, 2014.

[34] T. Alhmiedat, \& S. Yang. "A Survey: Localization and Tracking Mobile Targets Through Wireless Sensor Network", Proceedings of the PGNet Conference, Liverpool, UK, pp. 48, Jun. 2007.

[35] T. Alhmiedat, A. Abu Taleb, \& M. Bsoul. "A Study on Threats Detection and Tracking for Military Applications Using WSNs", International Journal of Computer Applications 40(15):12-18, February 2012.

https://doi.org/10.5120/5055-7347

[36] Janus Jade A. Basa\& Patrick Lourenz G. Cu, "Smart Inventory Management System for Photovoltaic-Powered Freezer Using Wireless Sensor Network", International 
Amer Abu Salem et al., International Journal of Emerging Trends in Engineering Research, 8(5), May 2020,2126 - 2132

Journal of Emerging Trends in Engineering Research, Vol 7, No. 10, 2019.

https://doi.org/10.30534/ijeter/2019/057102019

[37] Jojo F. Blanza1 , Lawrence Materum, "Grouping of COST 2100 Indoor Multipaths Using Simultaneous Clustering and Model Selection”, International Journal of Emerging Trends in Engineering Research, Vol 7, No. 8, 2019

https://doi.org/10.30534/ijeter/2019/15782019 